\section{Preditores de sucesso da metformina no tratamento do diabetes mellitus gestacional}

\section{Predictors of success of metformin treatment of diabetes mellitus in pregnancy}

Jean Carl Silva 1

Bárbara Vicente de Souza 2

Mariana Ribeiro e Silva 3
1-3 Departamento de Obstetrícia. Universidade da Região de Joinville. Rua Paulo Malschitzki, 10. Zona Industrial Norte. Joinville, SC, Brasil. CEP: 89.219-710. E-mail: jean.carl@univille.br

\begin{abstract}
Objectives: to identify characteristics of the mother related to failure of treatment of diabetes mellitus in pregnancy using metformin.

Methods: an observational retrospective study (July 2008-September 2010), with 104 pregnant women with pregnancy-related diabetes mellitus needing therapy to complement dietary treatment and using metformin. The women were divided into two groups: successful treatment $(n=82)$, where the drug was maintained up to delivery; treatment failure $(n=22)$ where the drug was replaced by insulin. The maternal variables studied were age, number of deliveries, weight gain during pregnancy, fasting glycaemia and two hours after OGTT75g, gestational age on diagnosis of diabetes mellitus. Variables relating to the newborn were incidence of GIG and neonatal hypoglycaemia. The clinical and metabolic characteristics of the mother and perinatal results were compared. The level of significance used was $5 \%$.

Results: there were diferences in the gestational age on diagnosis of diabetes melittus (27.5 vs 24.3 weeks; $p=0.02)$, body mass index (BMI) (27.5 vs 31.6 $\left.\mathrm{kg} / \mathrm{m}^{2} ; p=0.01\right)$, and fasting glycaemia on $O G T T 75 \mathrm{~g}$ (91.5 vs $108 \mathrm{mg} / \mathrm{dL} ; p=0.02)$, in the treatment success and failure groups, respectively. Treatment failure increased the weight index of newborns $(2.8 \mathrm{vs} 3$ $\left.\mathrm{g} / \mathrm{cm}^{3} ; p=0.05\right)$, the incidence of $G I G$ in RNs (4.9 vs $22.7 \%$; $p=0.02)$ and neonatal hypoglycaemia (6.1 vs $18.2 \% ; p=0.04)$. The data on newborns were secondary objectives.

Conclusions: early diagnosis, higher BMI and higher fasting glycaemia OGTT75g were more related to treatment failure.
\end{abstract}

Key words Diabetes, gestational, Metformin, Infant, newborn.

\section{Resumo}

Objetivos: identificar caracteristicas maternas relacionadas à falha no tratamento do diabetes mellitus gestacional (DMG) com metformina.

Métodos: estudo retrospectivo observacional (julho/2008-setembro/2010), com 104 gestantes com DMG que necessitaram de terapêtica complementar a dietoterapia e utilizaram metformina. Dividiram-se em dois grupos: sucesso terapêutico $(n=82)$, onde a droga foi mantida até o parto; outro com falha terapêutica $(n=22)$ onde a droga foi substituida por insulina. Variáveis maternas estudadas: idade, número de partos, ganho de peso na gestação, glicemia de jejum (GJ) e $2 \mathrm{~h}$ no OGTT75g, idade gestacional de diagnóstico de DMG (IGDMG). Variáveis do recém nascido: incidência de RNs GIG e hipoglicemia neonatal. Foram comparadas as características clínico-metabólicas maternas e os resultados perinatais, o nivel de significância utilizado foi de $5 \%$.

Resultados: apresentaram diferença na IGDMG (27,5 vs 24,3 semanas; $p=0,02)$, no índice de massa

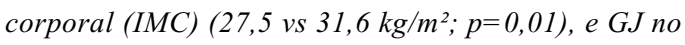
OGTT75g (91,5 vs $108 \mathrm{mg} / \mathrm{dL}$; $p=0,02)$, nos grupos de sucesso e falha respectivamente. A falha terapêutica aumentou o indice ponderal dos RNs $(2,8 \mathrm{vs} 3$ $\left.\mathrm{g} / \mathrm{cm}^{3} ; \mathrm{p}=0,05\right)$, incidência de RNs GIG (4,9 vs 22,7\%; $p=0,02)$ e hipoglicemia neonatal (6,1 vs $18,2 \%$; $p=0,04)$. Os dados dos RNs foram objetivos secundários.

Conclusões: o diagnóstico precoce, o maior IMC e maior GJ OGTT75g estiveram mais relacionados a falha terapêtica.

Palavras-chave Diabetes gestacional, Metformina, Recém-nascido 


\section{Introdução}

As mulheres que desenvolvem diabetes mellitus gestacional (DMG) compartilham muitas características metabólicas com as do diabetes mellitus tipo 2 (DM2). Estudos mostram alterações pós-parto em mulheres que desenvolveram DMG, como defeitos na secreção de insulina e diminuição na sensibilidade à insulina, alterações típicas do DM. ${ }^{1}$ Alguns autores vêem as duas entidades clínicas, como a mesma doença, porém com diferentes nomes, e representada por uma contínua deteriorização da tolerância a glicose, partindo do DMG para a intolerância a glicose e para o DM2.1,2 A metformina, droga de primeira escolha no tratamento do DM2, age diminuindo a gliconeogênese hepática, a absorção da glicose e aumentando a utilização periférica da glicose. Esta droga tem indicação no DMG por ser este metabolicamente semelhante ao DM2. A metformina atravessa a barreira placentária, porém em série de casos, quando foi utilizada em ovários policísticos, durante o primeiro trimestre, não apresentou efeitos teratogênicos.1,3

Os primeiros estudos relacionando diabetes, gestação e agentes hipoglicemiantes foram realizados em gestantes portadoras de DM2 com valores glicêmicos mais liberais mascarando a verdadeira causa das anormalidades fetais e neonatais, se a hiperglicemia ou a ação dos hipoglicemiantes orais. ${ }^{4}$

Em um estudo randomizado, controlado, com uma casuística de 751 gestantes, com o mesmo resultado perinatal do grupo randomizado para insulinoterapia, quando feita análise qualitativa da utilização da metformina encontrou-se como resultado que as gestantes preferem terapêutica oral em vez da insulinoterapia. 5 A taxa de sucesso com o tratamento com metformina varia entre $53 \% 5$ e $75 \%{ }^{6}$ e as características populacionais ou metodológicas podem justificar estas diferenças.

Além da melhor aceitação, a terapêutica oral teria um impacto nos custos do tratamento do DMG, que é responsável por grande parte da verba direcionada a saúde pública, ${ }^{7}$ assunto de extrema importância para países subdesenvolvidos.

Avaliar as características clínico-metabólicas das gestantes para identificar um grupo mais suscetível a falha na terapêutica com metformina é o objetivo deste estudo.

\section{Métodos}

Foi realizado um estudo retrospectivo descritivo em parte de uma população que participou de um ensaio clínico randomizado (comitê de ética no 037/10). A amostra foi composta pelas gestantes com diagnóstico de DMG alocadas no grupo tratado com metformina. As gestantes foram divididas em dois grupos: um onde a droga foi mantida até o momento do parto, com o controle glicêmico adequado sendo considerado sucesso terapêutico $(n=82)$. Na falta de controle glicêmico ao atingir a dose máxima, a terapêutica foi substituída para insulinoterapia e considerada falha terapêutica $(n=22)$. Os níveis glicêmicos considerados adequados foram glicemia de jejum $90 \mathrm{mg} / \mathrm{dL}$ e uma hora pós prandial 120 $\mathrm{mg} / \mathrm{dL}$. Foram comparadas as características clínicometabólicas maternas.

A pesquisa foi conduzida de acordo com as regulamentações da Resolução 196/96 do Conselho Nacional de Saúde, e foi realizada após autorização da Comissão de Ética e Pesquisa da Universidade da Região de Joinville e dos hospitais, sob número 037/10. Os pesquisadores não possuem vínculo com fabricantes de medicamentos ou equipamentos citados no estudo nem conflito de interesses. As gestantes leram e assinaram o termo de consentimento livre e esclarecido.

O estudo foi realizado na Maternidade Darcy Vargas (MDV), no Centro Hospitalar UNIMED e Hospital Dona Helena (HDH), no período de $1^{\circ}$ de Julho de 2008 a 30 de outubro de 2010. Os serviços possuem atendimento multidisciplinar para as gestantes portadoras de diabetes mellitus, contando com nutricionista, fisioterapeuta, psicóloga, enfermeira, obstetra e endocrinologista que fizeram o acompanhamento quinzenal das gestantes. A dieta foi calculada conforme peso e idade gestacional oscilando entre 25 e $35 \mathrm{kcal} / \mathrm{kg}$ nas pacientes obesas e com peso adequado respectivamente, fracionada em três refeições e quatro lanches, com 35 a $45 \%$ das calorias de carboidratos.

Foram selecionadas gestantes com diagnóstico de DMG conforme critérios da Organização Mundial da Saúde $^{8}$ que necessitaram terapêutica complementar a dietoterapia e à atividade física para controle glicêmico, com idade mínima de 18 anos, idade gestacional entre 11 e 33 semanas, gestação única, circunferência abdominal fetal no percentil normal $(>10 \%$ e $<75 \%)$ e ausência de outras patologias que interferiram nos resultados perinatais ou na terapêutica hipoglicêmica. Foram excluídas as gestantes com intolerância as drogas, desejo da paciente, presença de risco para o concepto (Circunferência abdominal fetal PCT $>97 \%$ ou $<5 \%$ ), perda de seguimento e óbito fetal.

As gestantes realizaram um perfil glicêmico domiciliar, com glicemia capilar periférica (GCP) 
após sete dias da orientação inicial, com determinação da glicemia em jejum, uma hora após o café, almoço e jantar. Os valores aceitáveis de glicemia foram $90 \mathrm{mg} / \mathrm{dL}$ para o jejum e $120 \mathrm{mg} / \mathrm{dL}$ para o pós-prandial, se dois pontos estivessem alterados, foi oferecida a participação no estudo. ${ }^{9}$ As pacientes selecionadas coletaram GCP domiciliar em jejum e pós-prandial para ajuste da dose da medicação.

A metformina foi utilizada na dose inicial de 500 mg no café e jantar, sendo aumentada em 500 a 1000 mg quinzenalmente até o controle glicêmico ou até atingir a dose máxima de $2500 \mathrm{mg} / \mathrm{dia} .{ }^{7}$ Quando o controle glicêmico não foi atingido com a dose máxima, a terapêutica foi substituída pela insulinoterapia na dose de $0,7 \mathrm{UI} / \mathrm{kg} / \mathrm{dia}$ com doses de insulina regular pré-prandial e NPH (Neutral Protamine de Hagedorn) ao deitar. 10 A metformina e a insulina foram fornecidas gratuitamente pela rede pública de saúde.

As variáveis maternas avaliadas foram: idade, paridade, índice de massa corporal, ganho de peso durante a gestação, idade gestacional no momento do diagnóstico (calculada pela primeira ultrassonografia realizada pela paciente), tipo de tratamento (metformina ou insulina), doses utilizadas no tratamento, necessidade de troca de terapêutica, nível glicêmico materno durante o pré-natal, hemoglobina glicosilada (pelo método de imunoturbimetria), idade gestacional de término da gravidez, hipoglicemias severas que necessitaram internação, via de parto, além de outras intercorrências.

As variáveis do recém-nascido avaliadas foram: peso ao nascer, adequação do peso ao nascer, em especial os recém-nascidos grandes para a idade gestacional (GIG) (peso acima do percentil $90 \mathrm{em}$ curvas de crescimento),11 índice ponderal $\left(\right.$ peso/altura $\left.{ }^{3}\right)$, presença de hipoglicemia fetal $(<40$ $\mathrm{mg} / \mathrm{dL}), \mathrm{GCP}$ na $1^{\mathrm{a}}, 3^{\mathrm{a}}$ e $6^{\mathrm{a}}$ hora pós-nascimento, número de dias de internação, necessidade de UTI neonatal, óbito e condições de alta.

Os desfechos foram analisados descritivamente com cálculo de médias, desvios-padrão, frequências absolutas e relativas. Para a análise da hipótese de igualdade entre a média dos dois grupos foram utilizados o teste $t$ de Student e o teste "U" de MannWhitney. Para se testar a homogeneidade dos grupos em relação às proporções foi utilizado o teste quiquadrado ou o teste exato de Fisher. O nível de significância utilizado para os testes foi de $5 \%$. Foi realizada análise multivarida para determinar o fator de maior relação com o desfecho sendo que as variáveis foram alocadas para análise por nível de associação, $p<0,05$. Através da curva ROC foram obtidos os pontos de corte para as variáveis com $p<0,05$, e cálculo do odds ratio (OR), sensibilidade e especificidade.

\section{Resultados}

Foram selecionadas 108 gestantes que aceitaram participar do ensaio. Houve perda de seguimento em duas, troca da medicação em uma por intolerância a droga, e ocorreu um óbito intra-útero. No total foram avaliadas 104 gestantes, sendo $82(78,8 \%)$ no grupo que obteve sucesso terapêutico e $22(21,2 \%)$ no grupo com falha na terapêutica.

Algumas características clínico-metabólicas das gestantes não apresentaram diferença no grupo de sucesso e falha respectivamente, são estas: idade materna $(32,42$ vs 32,38 semanas; $p=0,96)$, primiparidade $(20,7$ vs $4,5 \% ; p=0,11)$, ganho de peso durante a gestação $(7,30$ vs $5,38 \mathrm{~kg} ; p=0,15)$, glicemia de 2 horas no OGTT75g (164 vs 167 $\mathrm{mg} / \mathrm{dL} ; p=0,99)$.

A idade gestacional no momento do diagnóstico teve valores maiores no grupo de sucesso. Foi encontrada diferença estatística $(27,57$ vs 24,33 semanas; $p=0,02$ ), com área sob a curva ROC de 0,655 com valor de corte de 26,5 semanas mostrando uma sensibilidade de $68,3 \%$ e uma especificidade de $45,5 \%$. O odds ratio foi de 2,07 (IC95\%: 0,97-4,36).

$\mathrm{O}$ índice de massa corporal materno foi menor no grupo com sucesso $\left(27,57\right.$ vs $31,61 \mathrm{~kg} / \mathrm{m}^{2}$; $p=0,01)$, com área sob a curva ROC de 0.677 encontrando-se um ponto de corte de $27,5 \mathrm{~kg} / \mathrm{m}^{2} \mathrm{com}$ uma sensibilidade de $72,7 \%$ e especificidade de $47,6 \%$ e odds ratio 1,24 (IC95\%: 1,01-1,51) (Figura 1).

\section{Figura 1}

Curva ROC com sensibilidade e especificidade para o índice de massa corporal.

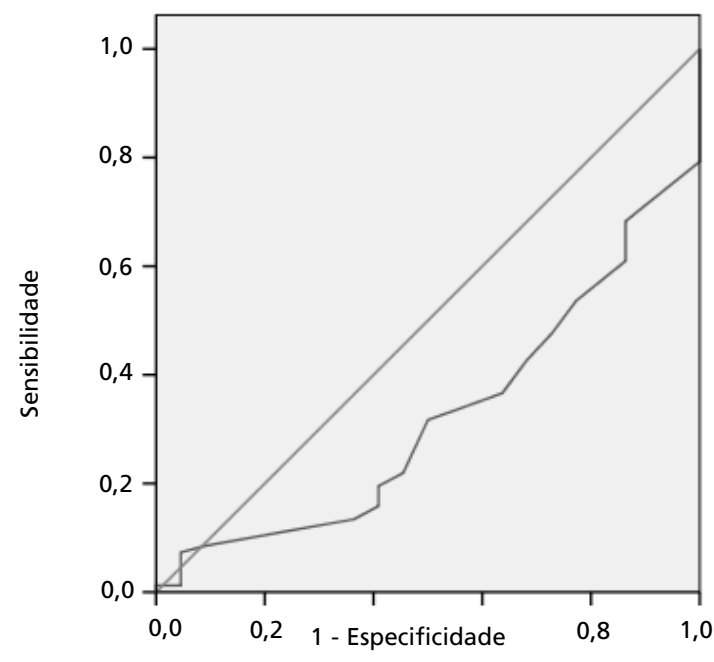

Sensibilidade $=72,7 \%$; Especificidade $=47,6 \%$. 
A glicemia em jejum no OGTT75g também foi menor no grupo de sucesso $(91,56$ vs 108,09 mg/dL; $p=0,02$ ), com área sob a curva ROC de 0,661 foi encontrado um ponto de corte de $92,5 \mathrm{mg} / \mathrm{dL}$ com uma sensibilidade de $68,2 \%$ e um especificidade de $41,9 \%$ e odds ratio 1,28 (IC95\%: 1,01-1,60). Sendo esta a variável com maior relação com o sucesso terapêutico.

A intercorrência materna mais frequente foi a infecção urinária em oito gestantes (7,7\%). Nenhuma paciente necessitou internação hospitalar para controle de hipoglicemia.

Quanto ao controle glicêmico encontramos valores menores nas glicemias pré $(88,17$ vs 98,0 $\mathrm{mg} / \mathrm{dL} ; p=0,01)$ e 1 hora pós-prandial $(122,58$ vs $138,62 \mathrm{mg} / \mathrm{dL} ; p<0,01)$ no grupo de sucesso, mas sem diferença na hemoglobina glicosilada fração A1C (HBA1C) do $3^{\circ}$ trimestre $(5,57$ vs 5,53\%; $p=1,00)$ nos grupos de sucesso e falha respectivamente.

$O$ resultado perinatal não apresentou diferença na via de parto, com taxa de cesarianas $(68,3$ vs $54,4 \% ; p=0,23)$, na idade gestacional no momento do parto $(38,4$ vs 37,7 semanas; $p=0,06)$, no peso médio do recém-nascido (RN) (3133 vs 3363 g; $p=0,07)$, presença de RNs pequenos para idade gestacional $(3,7$ vs $4,5 \%)$, apgar menor que 7 no primeiro $(4,8 v s 7,7 \% ; p=1,00)$ e quinto minuto $(0 v s$ $2,6 \% ; p=1,00)$, nos grupos de sucesso e falha respectivamente.

Nas glicemias capilares coletadas na $1^{\text {a }}$ hora (61,2 vs 53,7 mg/dL; $p=0,17), 3^{\text {a }}$ hora (62 vs 58 $\mathrm{mg} / \mathrm{dL} ; p=0,91)$ e $6^{\text {a }}$ hora pós-nascimento $(59,4 v s$ $57,2 \mathrm{mg} / \mathrm{dL} ; p=0,43)$, além da necessidade de tratamento em unidade de terapia intensiva (UTI) $(6,1 \mathrm{vs}$ $18,2 \% ; p=0,07)$ também não foi encontrada diferença, nos grupos de sucesso e falha respectivamente. O desconforto respiratório foi a principal causa de ingresso em UTI.

No índice ponderal dos RNs $\left(2,83\right.$ vs $2,99 \mathrm{~g} / \mathrm{cm}^{3}$; $p=0,05)$, na presença de RNs grandes para a idade gestacional $(4,9$ vs $22,7 \%$; $p=0,02)$, e na presença de hipoglicemia neonatal $(6,1$ vs $18,2 \% ; p=0,04)$ nos grupos de sucesso e falha respectivamente foi encontrado diferença.

O óbito intraútero ocorreu em um gestante com glicemias normais, com peso fetal adequado para idade gestacional, sendo encaminhado para necropsia com resultado não conclusivo. Não houve caso de malformação fetal.

As características gerais e metabólicas das gestantes, além das características dos recém nascidos estão agrupados na Tabela 1 .

\section{Discussão}

Neste estudo houve diferença significativa na idade gestacional no momento do diagnóstico (27,5 vs 24,3 semanas), no índice de massa corporal (27,5 vs 31,6 $\left.\mathrm{kg} / \mathrm{m}^{2}\right)$, e na glicemia de jejum no OGTT75g $(91,5$ vs $108 \mathrm{mg} / \mathrm{dL}$ ), nos grupos de sucesso e falha na terapêutica respectivamente. A falha na terapêutica com metformina aumentou significantemente o índice ponderal dos recém-nascidos (RNs) $(2,83 v s$ $\left.2,99 \mathrm{~g} / \mathrm{cm}^{3}\right)$, a incidência de RNs GIG (4,9 vs 22,7\%) e da hipoglicemia neonatal $(6,1$ vs $18,2 \%)$.

A taxa de sucesso na terapêutica com a metformina nas gestantes portadoras de DMG em ensaios clínicos randomizados varia entre $53 \mathrm{e}$ $75 \% .5,12,13$ Nós encontramos $78 \%$ de sucesso terapêutico. Sendo a glibenclamida o outro hipoglicemiante oral mais estudado em comparação com a insulinoterapia, esta apresenta taxas de sucesso semelhantes oscilando entre $58 \%$ e $96 \% .14$ 17

O grupo que obteve sucesso na terapêutica com a metformina, não sendo necessária a troca ou adição de insulina ao tratamento, era composto de pacientes com idade gestacional no momento do diagnóstico mais avançada, menor índice de massa corporal e valores menores na glicemia em jejum no teste de diagnóstico. Em um estudo de coorte de cinco anos com a glibenclamida foram encontradas as mesmas características relacionadas ao sucesso terapêutico quanto a idade gestacional no momento do diagnóstico e no índice de massa corporal; 18 outros autores encontraram a mesma relação com a glicemia em jejum no OGTT75g. 19

A glicemia de jejum no OGTT75g foi a variável com maior relação à falha, com um valor de corte de $92,5 \mathrm{mg} / \mathrm{dL}$, obtido através da curva ROC, apresentando um OR baixo, 1,28 (IC95\%: 1,01-1,60). Os valores de sensibilidade e especificidade para esta variável neste estudo foram $68,2 \%$ e $41,9 \%$ respectivamente. Outros autores estudando a glibenclamida encontrou uma sensibilidade de $98 \%$ e especificidade de $65 \%$ para sucesso na terapêutica avaliando uma associação entre a glicemia (glicemia em jejum $<110$ e pós-prandial de $<140 \mathrm{mg} / \mathrm{dL}$ ) e a idade gestacional $(<30$ semanas $)$ no momento da falha na dietoterapia. ${ }^{16}$ É possível que esses autores tenham encontrado níveis mais altos de sensibilidade e especificidade pelo estudo ter níveis aceitáveis mais elevados de glicemia de jejum e pós prandial que no presente estudo.

Quanto ao controle glicêmico encontramos valores menores nas glicemias pré e uma hora pósprandial nas pacientes que obtiveram sucesso com a 
Características gerais e metabólicas das gestantes e dos recém nascidos.

\begin{tabular}{|c|c|c|c|}
\hline Gestantes & $\begin{array}{l}\text { Sucesso } \\
(\mathrm{N}=82)\end{array}$ & $\begin{array}{l}\text { Falha } \\
(\mathrm{N}=22)\end{array}$ & $p$ \\
\hline Idade (anos) $\bar{X}(D P)$ & $32,42(5,65)$ & $32,38(6,13)$ & 0,96 \\
\hline Primiparidade $\mathrm{n}(\%)$ & $17(20,7 \%)$ & $1(4,5 \%)$ & 0,11 \\
\hline IG dignóstico (semanas) $\bar{X}(D P)$ & $27,57(6,29)$ & $24,33(6,68)$ & 0,02 \\
\hline IMC $\bar{X}(D P)$ & $27,64(5,64)$ & $31,62(5,07)$ & 0,01 \\
\hline $\mathrm{G}$ peso $(\mathrm{kg}) \bar{X}(\mathrm{DP})$ & $7,30(8,16)$ & $5,38(4,46)$ & 0,15 \\
\hline OGTT jejum (mg/dL) $\bar{X}(D P)$ & $91,56(14,90)$ & $108,09(37,55)$ & 0,02 \\
\hline OGTT 2 horas $(\mathrm{mg} / \mathrm{dL}) \bar{X}(\mathrm{DP})$ & $164,15(20,80)$ & $167,15(31,28)$ & 0,99 \\
\hline $\mathrm{G}$ jejum (mg/dL) $\bar{X}(D P)$ & $88,17(9,75)$ & $98,00(21,29)$ & 0,01 \\
\hline $\mathrm{G} 1 \mathrm{~h}(\mathrm{mg} / \mathrm{dL}) \bar{X}(\mathrm{DP})$ & $122,58(18,42)$ & $138,62(26,22)$ & $<0,01$ \\
\hline $\mathrm{HbA1C} 3 \mathrm{~T} \quad \overline{\mathrm{X}}(\mathrm{DP})$ & $5,57(0,97)$ & $5,53(0,74)$ & 1,00 \\
\hline \multicolumn{4}{|l|}{ Recém-nascidos } \\
\hline Cesarianas $\mathrm{n}(\%)$ & $56(68,3 \%)$ & $12(54,5 \%)$ & 0,23 \\
\hline IG parto $\bar{X}(D P)$ & $38,37(1,30)$ & $37,7(1,69)$ & 0,06 \\
\hline Peso RN (g) $\bar{X}(D P)$ & $3133,16(489,31)$ & $3363,50(647,33)$ & 0,07 \\
\hline Índice ponderal $\bar{X}(D P)$ & $2,83(0,48)$ & $2,99(0,33)$ & 0,05 \\
\hline GIG $n(\%)$ & $4(4,9 \%)$ & $5(22,7 \%)$ & 0,02 \\
\hline PIG $n(\%)$ & $3(3,7 \%)$ & $1(4,5 \%)$ & 1,00 \\
\hline Apgar $1<7 \quad \mathrm{n}(\%)$ & $1(4,8 \%)$ & $6(7,7 \%)$ & 1,00 \\
\hline Apgar $5<7 \quad n(\%)$ & $0(0 \%)$ & $2(2,6 \%)$ & 1,00 \\
\hline GCP $1 \mathrm{~h}(\mathrm{mg} / \mathrm{dL}) \overline{\mathrm{X}}(\mathrm{DP})$ & $61,20(15,65)$ & $53,73(15,10)$ & 0,17 \\
\hline $\mathrm{GCP} 3 \mathrm{~h}(\mathrm{mg} / \mathrm{dL}) \overline{\mathrm{X}}(\mathrm{DP})$ & $62,06(20,25)$ & $58,05(13,60)$ & 0,91 \\
\hline GCP $6 \mathrm{~h}(\mathrm{mg} / \mathrm{dL}) \bar{X}(\mathrm{DP})$ & $59,38(11,57)$ & $57,21(9,36)$ & 0,43 \\
\hline Hipoglicemia n (\%) & $6(7,31 \%)$ & $5(22,72 \%)$ & 0,04 \\
\hline UTI n (\%) & $5(6,09 \%)$ & $4(18,18 \%)$ & 0,07 \\
\hline
\end{tabular}

$\mathrm{DP}=$ desvio-padrão; IG= idade gestacional em semanas completas; $I M C=$ índice de massa corporal; $\mathrm{G}$ peso=ganho de peso durante a gestação $(\mathrm{kg})$; OGTT= teste de tolerância oral a glicose $(\mathrm{mg} / \mathrm{dL})$; IG parto= idade gestacional no momento do parto (semanas); Peso $\mathrm{RN}=$ peso do recém-nascido (gramas); $\mathrm{GIG}=$ grande para a idade gestacional; Apgar $1=$ Apgar no $1^{\circ}$ minuto; Apgar $5=$ Apgar $5^{\circ}$ minuto; GCP $1 \mathrm{~h}, 3 \mathrm{~h}$ e $6 \mathrm{~h}=$ glicemia capilar periférica na $1^{\mathrm{a}}, 3^{\mathrm{a}}$ e $6^{\mathrm{a}}$ hora após o nascimento; Hipoglicemia= hipoglicemia pós-parto $(<40 \mathrm{mg} / \mathrm{dL})$; UTI= necessidade de internação em unidade de terapia intensiva; G jejum= média das glicemias em jejum $(\mathrm{mg} / \mathrm{dL}) ; \mathrm{G} 1 \mathrm{~h}=$ glicemia média 1 hora após as refeições $(\mathrm{mg} / \mathrm{dL}) ; \mathrm{HBA} 1 \mathrm{C} 3 \mathrm{~T}=$ hemoglobina glicosilada fração A1C no $3^{\circ}$ trimestre $(\%)$.

metformina, sendo este o principal motivo para substituição da droga pela insulinoterapia, pois apenas uma paciente solicitou a troca por intolerância à droga. A hemoglobina glicosilada coletada no terceiro trimestre não apresentou diferença.

Outros autores que realizaram um estudo de coorte encontraram na idade gestacional no momento do diagnóstico ( $<25$ semanas) a variável com maior OR $(8,3)$ para falha na terapêutica com a glibenclamida. ${ }^{19}$ Neste estudo foi encontrado para idade gestacional menor que 26,5 semanas um OR 2,07 (IC95\%: 0,97-4,36). O índice de massa corpórea materno foi maior no grupo com falha, com um OR 1,24 (IC95\%: 1,01-1,51). O índice de massa corpórea materno foi maior no grupo com falha, com um OR 1,24 (IC95\%: 1,01-1,51). Enquanto alguns autores também encontraram igual importância nesta variável em estudos com a glibenclamida, 18 outros não encontraram essa relação, mas sim com maior idade materna e a multiparidade. 19 As características das mulheres com falha a glibenclamida sugerem que elas sejam mais propensas a ter intolerância à glicose previamente à gestação e serem menos propensas a responder a um secretagogo de insulina do que as mulheres que tiveram sucesso.

As taxas de sucesso e a identificação do grupo com maior risco para falha terapêutico com os hipoglicemiantes orais são de extrema importância, porém os resultados perinatais se sobrepujam a 
estes. A falha na terapêutica neste estudo, refletida na diferença das glicemias durante o tratamento, comprometeu o resultado perinatal aumentando o índice ponderal dos RNs, a incidência de RNs GIG e da hipoglicemia neonatal, resultado que é a favor da literatura ${ }^{20} \mathrm{Na}$ mesma análise com a glibenclamida não foi encontrado este impacto negativo sobre os RNs. ${ }^{16,18}$

Identificar precocemente o grupo com maior risco de falha na terapêutica com hipoglicemiantes orais pode minimizar o impacto sobre os resultados perinatais. ${ }^{19} \mathrm{Em}$ nosso estudo todos os filhos de mães com DMG que foram expostas a metformina tiveram maiores medidas de gordura subcutânea, mas a gordura corporal total foi a mesma das crianças cujas mães foram tratadas apenas com insulina. Estudos em longo prazo são necessários

\section{Referências}

1. Langer O. Oral hypoglycemic agents in pregnancy: their time has come. J Matern Fetal Neonatal Med. 2002; 12: 376-83.

2. Landon M, Gabbe S. Diabetes Mellitus. In: Barron W, Lindhemeir M, editors. Medical complications in pregnancy. Porto Alegre: Artes Médicas; 1996. p. 55-77.

3. Nanovskaya, T N, Nekkhayeva IA, Patrikeeva SL, Hankins GD, Ahmed MS. Transfer of metformin across the dually perfused human placental lobule. Am J Obstet Gynecol. 2006; 195: 1081-5

4. Stage E, Ronneby H, Damm P. Lifestyle change after gestational diabetes. Diab Res Clin Pract. 2004; 63: 67-72.

5. Sutherland H, Bewsher P, Cormack J, Hughes C, Russell G Stowers J. Effect of moderate dosage of chlorpropamide in pregnancy on fetal outcome. Arch Dis Child. 1974; 49: 283-91.

6. Rowan JA, Hague WM, Gao W, Battin MR, Moore MP Metformin versus insulin for the treatment of gestational diabetes. N Engl J Med. 2008; 358: 2003-15.

7. Silva JC, Pacheco C, Bizato J, De Souza BV, Ribeiro TE, Bertini AM. Metformin compared with glyburide for the management of gestational diabetes. Int J Gynaecol Obstet. 2010; 111: 37-40.

8. Chen Y, Quick WW, Yang W, Zhang Y, Baldwin A, Moran J, Moore V, Sahai N, Dall TM: Cost of gestational diabetes mellitus in the United States in 2007. Popul Health Manag. 2009; 12: 165-74.

9. Schmidt MI, Duncan BB, Reichelt AJ, Branchtein L, Matos MC, Costa e Forti A, Spichler ER, Pousada JMDC, Teixeira MM, Yamashita T. Gestational diabetes mellitus diagnosed with a 2-h 75-g oral glucose tolerance test and adverse pregnancy outcomes. Diabetes Care. 2001; 24: 1151-5.

10. Bertini AM, Taborda,W, Silva JC, Konz CD. Real target of $2 \mathrm{H}$ postprandial, glucose levels in order to prevent GIG? Macrosomic babies. J Perinat Med. 2005; 33: 298 para avaliar se esses achados persistem tardiamente durante a vida e se as crianças expostas a metformina desenvolverão menos gordura visceral e maior sensibilidade insulínica. ${ }^{21}$ Estudos sobre o desenvolvimento neuropsicomotor dos RNs a longo prazo se fazem necessários para validar o uso dos hipoglicemiantes orais durante a gestação. 22

De acordo com a metodologia realizada o estudo tem validade interna, porém mais estudos são necessários para que esses resultados possam ser utilizados para a população geral.

Percebe-se que é importante a identificação das características clínico-metabólicas maternas de falha na terapêutica com metformina precocemente para que consequentemente possam ser reduzidas as complicações fetais.

11. Silva J, Scheidt M, Heinen A. Diabete melito gestacional dose ideal de insulina utilizada durante o terceiro trimestre de gestação e resultados perinatais. Arq Catarinenses. 2008; 37: 76-80.

12. Lubchenco LO, Koops BL. Assessment of weight and gestational Age. In: Avery G. Neonatology: pathophysiology and management of the newborn. Philadelphia: Ed. J.B. Lippincott CO; 1987; p. 235-57.

13. Moore LE, Clokey D, Rappaport VJ, Curet LB. Metformin compared with glyburide in gestational diabetes: a randomized controlled trial. Obstet Gynecol. 2010; 115: 55-9.

14. Silva JC, Pacheco C, Bizato J, Souza BV, Ribeiro TE, Bertini AM. Metformin compared with glyburide for the management of gestational diabetes. Int J Gynaecol Obstet. 2010; 111: 37-40.

15. Bertini A, Silva J, Taborda W, Becker F, Bebber F, Viesi J, Aquim G, Ribeiro TE. Perinatal outcomes and the use of oral hypoglycemic agents. J Perinat Med. 2005; 33: 51923

16. Carles G, Germain L, Alassas N, El GW, Dallah F, Daigre JL. Treatment of gestational diabetes with oral hypoglycemic agents. J Gynecol Obstet Biol Reprod. 2010; 39: $139-43$.

17. Chmait R, Dinise T, Moore T. Prospective observational study to establish predictors of glyburide success in women with gestational diabetes mellitus. J Perinatol. 2004; 10: 617-22.

18. Langer O, Conway DL, Berkus MD, Xenakis EMJ, Gonzales ORN. A comparison of glyburide and insulin in women with gestational diabetes mellitus. New Engl J Med. 2000; 343: 1134-8.

19. Silva JC, Heinen, A, Scheidt MB, Marcondes MA, Bertini AM. Tratamento do diabetes mellitus gestacional com glibenclamida: fatores de sucesso e resultados perinatais. Rev Bras Ginecol Obstet. 2007; 29: 555-60. 
20. Kahn BF, Davies JK, Lynch AM, Reynolds RM, Barbour LA. Predictors of glyburide failure in the treatment of gestational diabetes. Obstet Gynecol. 2006; 107: 1303-9.

21. Rowan JA, Gao W, Hague WM, Mcintyre HD. Glycemia and its relationship to outcomes in the metformin in gestational diabetes trial. Diabetes Care. 2010; 33: 9-16.
22. Rowan J, Rush E, Obolonkin V, Battin M. Metformin in gestational diabetes: the offspring follow-up (MiG TOFU). Diabetes Care. 2011; 34: 2279-84.

Recebido em 17 de setembro de 2012

Versão final apresentada em 2 de janeiro 2013

Aprovado em 23 de abril de 2013 